\title{
Fracturas intraarticulares de radio distal: reducción bajo fluoroscopía versus asistencia artroscópica. Revisión sistemática
}

\author{
Distal radius intraarticular fractures: fluoroscopy reduction \\ versus arthroscopic assistance. Systematic review \\ Aguirre GL,* Cristiani WM,* Robles C,* Gutiérrez-Olivera N,* Paganini F,* Allende-Nores C* \\ Sanatorio Allende, Argentina.
}

RESUMEN. Introducción: Las fracturas intraarticulares de radio distal son un desafío terapéutico para el cirujano ortopedista. Hay estudios que avalan el uso de la fluoroscopía y otros promueven la artroscopia. Con este trabajo intentamos resumir la evidencia, para determinar si la asistencia artroscópica aporta beneficios adicionales para evitar incongruencias articulares en comparación con los resultados obtenidos bajo asistencia fluoroscópica. Material y métodos: Búsqueda sistemática de estudios prospectivos, retrospectivos, de cohortes, seguimiento, ensayos clínicos en PubMed, MEDLINE, Scopus, Scielo, Embase, Google Scholar y otras fuentes nacionales, incluyendo como palabras clave los términos: intraarticular distal radius fracture, wrist arthroscopy, arthroscopy, fluoroscopy. Los valores medios y desvíos estándar para cada característica, obtenidos de los trabajos seleccionados fueron analizados usando estadística descriptiva y gráficos ilustrativos. Resultados: Fueron evaluados 463 pacientes (256 mujeres y 207 hombres), con una edad promedio de 48.29 años y rango de 39 a 64 años. Los dos tratamientos (A y F) fueron homogéneos en cuanto a la edad de los pacientes que reportan ( $p=0.5820$ ) y el tiempo de seguimiento promedio ( $\mathrm{p}=0.9597)$. Sólo la desviación cubital y el DASH, para las cuales el grupo de artroscopía tuvo mejor desempeño, en las variables restantes las diferencias no fueron significativas. Conclusión: La evidencia disponible hasta la fecha es controvertida y no permite hacer recomendaciones a favor o en contra de estas intervenciones, encontrando otros factores que podrían influir en la toma de decisiones.

Palabras clave: Tratamiento, radio, distal, fluroscopía, artroscopía
ABSTRACT. Introduction: Intraarticular distal radius fractures are a therapeutic challenge for the orthopedist surgeon there are studies that support the use of fluoroscopy, and others promote arthroscopy, with this work we try to summarize the evidence, to determine whether arthroscopic assistance provides additional benefits to avoid joint incongruities compared to results obtained under fluoroscopic assistance. Material and methods: Systematic search for prospective, retrospective, cohort, follow-up, clinical trials on PubMed, MEDLINE, Scopus, Scielo, Embase, Google Scholar and other national sources, including as keywords the terms: «intra-articular distal radius fracture», «wrist arthroscopy», «arthroscopy», «fluoroscopy». The average values and standard offsets for each characteristic, obtained from the selected works, were analyzed using descriptive statistics and illustrative graphs. Results: 463 patients (256 women and 207 men) were evaluated, with an average age of 48.29 years and range from 39 to 64 years. The two treatments (A and F) were homogeneous in terms of the age of the patients reporting $(p=0.5820)$ and the average follow-up time $(p=0.9597)$. Only the ulnar deviation and DASH score, for which the arthroscopy group performed best, in the remaining variables the differences were not significant. Conclusion: The evidence available to date is conflicting, and does not allow recommendations to be made for or against these interventions, finding other factors that could influence decision-making.

Keywords: Treatment, radio, distal, fluroscopy, arthroscopy.

\section{Nivel de evidencia: IV}

* Servicio de Ortopedia y Traumatología, Sanatorio Allende, Córdoba (Capital). República de Argentina.

Correspondencia:

Gerardo Luis Aguirre

E-mail: gerardoaguirre_91@hotmail.com

Citar como: Aguirre GL, Cristiani WM, Robles C, Gutiérrez-Olivera N, Paganini F, Allende-Nores C. Fracturas intraarticulares de radio distal: reducción bajo fluoroscopía versus asistencia artroscópica. Revisión sistemática. Acta Ortop Mex. 2020; 34(6): 426-432. https://dx.doi.org/10.35366/99143

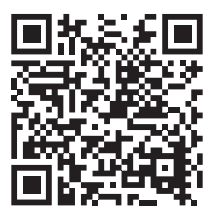




\section{Introducción}

Las fracturas intraarticulares de radio distal son un desafío terapéutico para el cirujano ortopedista, el objetivo final del tratamiento es lograr una reducción estable restaurando la anatomía articular. La evidencia es inconclusa en relación a qué material de osteosíntesis utilizar, pero existe consenso de que los pacientes adultos jóvenes que sufren una fractura desplazada inestable de radio distal se beneficiarán si se realiza tratamiento quirúrgico. ${ }^{1,2}$ Desde la publicación del trabajo de Knirk y Jupiter en 1986, corregir las incongruencias articulares se ha convertido en un factor crucial para la prevención del desarrollo de artrosis postraumática. ${ }^{3}$ Sin embargo, se considera que la relación entre la presencia de incongruencias articulares, el desarrollo de artrosis a largo plazo y los resultados funcionales deficientes en fracturas de radio distal no es lineal., ${ }^{4}$

La asistencia fluoroscópica es una herramienta indispensable para las cirugías traumatológicas, se utiliza en forma rutinaria para evaluar las superficies articulares ${ }^{6,7}$ y obtener una reducción anatómica. Sin embargo, al controlar los resultados postreducción mediante imágenes tomográficas se han observado incongruencias articulares infravaloradas por fluoroscopía. ${ }^{8}$

El desarrollo de la artroscopía de muñeca fue una progresión evolutiva del éxito de la aplicación de este método en otras articulaciones como rodilla y hombro. ${ }^{9}$ Su desarrollo ha sido considerable desde que Whipple y colaboradores realizaron la descripción original de esta técnica para la evaluación de la anatomía de la muñeca. ${ }^{9}$ Las indicaciones para la utilización de asistencia artroscópica en fracturas de radio distal incluyen la necesidad de examinar y evaluar las superficies condrales, la calidad de la reducción articular radial y de la articulación radio cubital distal, los ligamentos intercarpianos y el complejo del fibrocartílago triangular (CFCT). ${ }^{10}$

Algunos autores sugieren que el uso de asistencia artroscópica en fracturas intraarticulares de radio distal disminuye la incidencia de incongruencias articulares mayores de 2 $\mathrm{mm}$, no detectadas por fluoroscopía. $11,12,13,14$

El objetivo de este trabajo es realizar una revisión sistemática rigurosa de la bibliografía y metaanálisis que resuma la evidencia, con el fin de evaluar si la asistencia artroscópica en el tratamiento de fracturas intraarticulares de radio distal en adultos aporta beneficios adicionales para evitar incongruencias articulares en comparación con los resultados obtenidos mediante reducción indirecta sólo con asistencia fluoroscópica y si esto contribuiría en los resultados funcionales y el desarrollo de artrosis a largo plazo. Como objetivo secundario determinar en qué casos sería adecuado utilizar en forma rutinaria la asistencia artroscópica.

\section{Material y métodos}

Se llevó a cabo una revisión sistemática de la literatura y metaanálisis de los datos obtenidos siguiendo los criterios indicados por PRISMA (Preferred Reporting Items for Systematic Reviews and Meta-Analyses). ${ }^{15}$ Las bases de datos utilizadas para la búsqueda bibliográfica fueron PubMed, MEDLINE, LILACS, Scielo, Cochrane Library, Scopus, Embase, Google Scholar y páginas nacionales de Asociaciones, Bibliotecas específicas publicaciones periódicas no indexadas. Las palabras clave se registraron en la Tabla 1. Se estableció como límite de tiempo publicaciones realizadas entre 1990 y Mayo de 2017. Se seleccionaron estudios aleatorizados o no aleatorizados, prospectivos, retrospectivos, serie de casos, estudios de cohorte, estudios follow up y ensayos clínicos. Los idiomas seleccionados fueron español, inglés, francés y portugués.

En relación al tipo de participantes e intervención, se incluyeron artículos realizados en humanos de ambos sexos, mayores de 18 años, con fracturas de radio distal intraarticular tratadas por diferentes métodos de osteosíntesis (placas, tutor externo, clavijas de Kirschner percutáneas) que comparen pacientes en los que se utilizó sólo asistencia fluoroscópica para la reducción contra pacientes en quienes se empleó asistencia artroscópica. Se excluyeron trabajos que incluyeran participantes pediátricos, número de casos menor de 10, pacientes con fracturas extraarticulares. También se excluyeron cartas al editor, notas de autor, cursos de instrucción, notas técnicas, estudios experimentales (de intervenciones o prototipos, patentes, etcétera).

Un total de 361 artículos fue el resultado de la búsqueda utilizando las diferentes combinaciones de palabras clave. Dos autores (NG y GA) en forma independiente revisaron los artículos seleccionados. Se eliminaron los artículos duplicados. Ambos investigadores clasificaron los estudios según intervención y tipo de estudio a partir del título y resumen, realizando una segunda selección de artículos. En una tercera etapa a partir del texto completo de cada publicación se seleccionaron los artículos que cumplieran los criterios de inclusión en forma cualitativa y cuantitativa. Se registraron datos demográficos, tiempo de seguimiento, clasificación de la fractura, lesiones asociadas de tejidos blandos, tipo de osteosíntesis, escalas funcionales utilizadas, resultados objetivos, parámetros radiográficos y complicaciones.

Los valores medios y desvíos estándar para cada característica, obtenidos de los trabajos seleccionados, fueron analizados usando estadística descriptiva y gráficos ilustrativos. Se prefirieron los diagramas de cajas a los fines describir de manera robusta la distribución de cada variable.

Para comparar el desempeño de las técnicas se utilizaron modelos con distribución Gama, incluyendo un factor o covariable categórica que representa la técnica (siendo artroscopía como baseline) utilizada.

Las asociaciones fueron estimadas por medio de coeficientes de correlación, usando el coeficiente de Pearson para variables continuas y el de Spearman para correlaciones monótonas (no necesariamente lineales). 


\section{Resultados}

Luego de eliminar los artículos duplicados, el cribado dio como resultado 113 publicaciones, al analizar el texto completo de cada estudio se seleccionaron 82 con criterios de elegibilidad (Figura 1). Siete artículos cumplieron con los criterios de inclusión según tipo de estudio y participantes. En relación a la fecha de publicación sólo un artículo fue publicado entre 1990 y $1999,{ }^{14}$ un artículo entre 2000 y $2009,,^{12}$ cinco fueron publicados entre 2010 y Mayo de 2017. $11,16,17,18,19$

En cuanto al tipo de estudio, se incluyeron tres ensayos de cohorte prospectivo aleatorizado (Nivel de evidencia $\mathrm{II}+),{ }^{11,14,18}$ dos estudios de cohorte prospectivo no aleatorizado (Nivel de evidencia II -), ${ }^{12,17}$ un trabajo de cohorte no aleatorizado con un grupo prospectivo y otro retrospectivo (Nivel de evidencia II-) $)^{16} \mathrm{y}$ un estudio serie de casos retrospectivo (Nivel de evidencia III). ${ }^{19}$

Se evaluó un total de 463 pacientes pertenecientes a los siete estudios revisados, que incluyeron 256 mujeres y 207 hombres, con una edad promedio de 48.29 años, con un rango de 39 a 64 años.

El tiempo de seguimiento promedio fue de 15.98 meses, el rango varió entre tres y 31 meses en el estudio realizado por Christiaens y colaboradores, ${ }^{19}$ siendo el de menor seguimiento, con respecto al estudio realizado por Doi y su equipo, ${ }^{14}$ quienes presentaron un seguimiento promedio de 31 meses.

Seis trabajos ${ }^{11,12,14,16,18,19}$ utilizaron la clasificación AO/ ASIF $^{20}$ para radio distal; sin embargo, dos de ellos sólo nombran la clasificación, pero no desglosan el número de casos de cada tipo de fractura. ${ }^{11,12}$ Sólo en el trabajo realizado por Lalone y colaboradores ${ }^{17}$ se utilizó la clasificación McMurtry, ${ }^{20}$ que considera el número de fragmentos articulares sin valorar el desplazamiento de éstos, clasificación actualmente de poco uso.

Los métodos utilizados para la evaluación funcional fueron heterogéneos, la escala DASH ${ }^{21}$ (Disabilities of the Arm, Shoulder and Hand) fue la más elegida, seleccionada en cuatro trabajos, ${ }^{11,12,16,18}$ Kordasiewicz y colaboradores ${ }^{16}$ además emplearon la escala visual análoga para el dolor (EVA). Lalone y su equipo ${ }^{17}$ utilizaron la escala PRWE (Patient-Rated Wrist Evaluation), Doi y colegas Clasificación Funcional de Garltland y Werley. ${ }^{20}$

Sólo un trabajo evaluó el tiempo de reincorporación laboral. ${ }^{11}$
El registro de datos funcionales objetivos: fuerza de prensión, flexión, extensión, desviación radial, desviación cubital, pronación, supinación no fue uniforme. Lalone y colaboradores ${ }^{17}$ registraron sólo fuerza de prensión y Christiaens y su equipo ${ }^{19}$ no examinaron estas características.

La evaluación de parámetros radiográficos (inclinación radial, inclinación volar, varianza cubital) fue documentada en cinco trabajos. ${ }^{11,12,14,18,19}$ Sólo Doi y colaboradores ${ }^{14}$ y Kordasiewicz y colegas ${ }^{16}$ estudiaron el grado de artrosis mediante la Clasificación Kirk and Jupiter.

En relación a las complicaciones, el grupo artroscopía registró siete complicaciones, un caso de impactación cubital por mala consolidación radial asociado a varianza cubital positiva; cuatro casos de distrofia simpática refleja; un caso de síndrome del túnel carpiano; y un caso de artrosis radio cubital distal. El grupo fluoroscopía presentó 16 complicaciones, inestabilidad radio cubital distal en cuatro pacientes; tornillos intraarticulares en dos pacientes; distrofia simpática refleja en siete casos; síndrome del túnel carpiano en dos casos; y un caso de infección superficial en el sitio de clavijas.

Los trabajos científicos revisados indicaron que los dos tratamientos (A y F) fueron homogéneos en cuanto a la edad de los pacientes que reportan $(\mathrm{p}=0.5820)$ y el tiempo de seguimiento promedio $(\mathrm{p}=0.9597)$ bajo control de los mismos en los estudios.

La Tabla 2 muestra los valores medios y desvíos estándar para las principales características indagadas por los estudios en cada conjunto de pacientes y el valor-p, obtenido luego de la comparación de ambos tratamientos. Nótese que, salvo para la desviación cubital y el escore DASH para las cuales la reducción asistida por artroscopía tuvo mejor desempeño, en las variables restantes las diferencias entre ésta y la fluoroscopía no fueron significativas. Las Figuras 2 y 3 muestran las distribuciones para algunas de las variables que se describen en la Tabla 2 por tratamiento.

Otras características como incongruencia articular, cantidad de casos presentados según clasificación $\mathrm{AO} / \mathrm{ASIF}$, tipo de osteosíntesis mostraron en general elevada heterogeneidad en ambos tratamientos y valores promedio con escasa precisión. Además, varias de estas variables no fueron indagadas en todos los trabajos revisados. Se destaca, no obstante, la diferencia observada entre artroscopía y fluoroscopía en la cantidad de lesiones FCT (Tabla 2) con IC 95\%: 8.10, 16.55 para la primera.

arthroscopy-distal- radius- fracture-(fluoroscopy- review- sample study- follow up- retrospective study-cohort study-case series) arthroscopy-wrist (fluoroscopy-follow up-retrospective study-cohort study- case series- sample study)

Wrist- arthroscopy- (review-fluoroscopy review)

wrist-arthroscopy-intra-articular- fracture- of- the- distal- radius- (fluoroscopy/wrist)

Wrist- arthroscopy- intra-articular- distal- radius- fracture-(follow up- cohort study-sample study-retrospective study- case series- review- fluoroscopy review)

intra-articular- distal- radius- fracture-(fluoroscopy review- retrospective study- sample study- follow up- case series)

Arthroscopy- intra-articular- distal- radius- fracture-fluoroscopy 


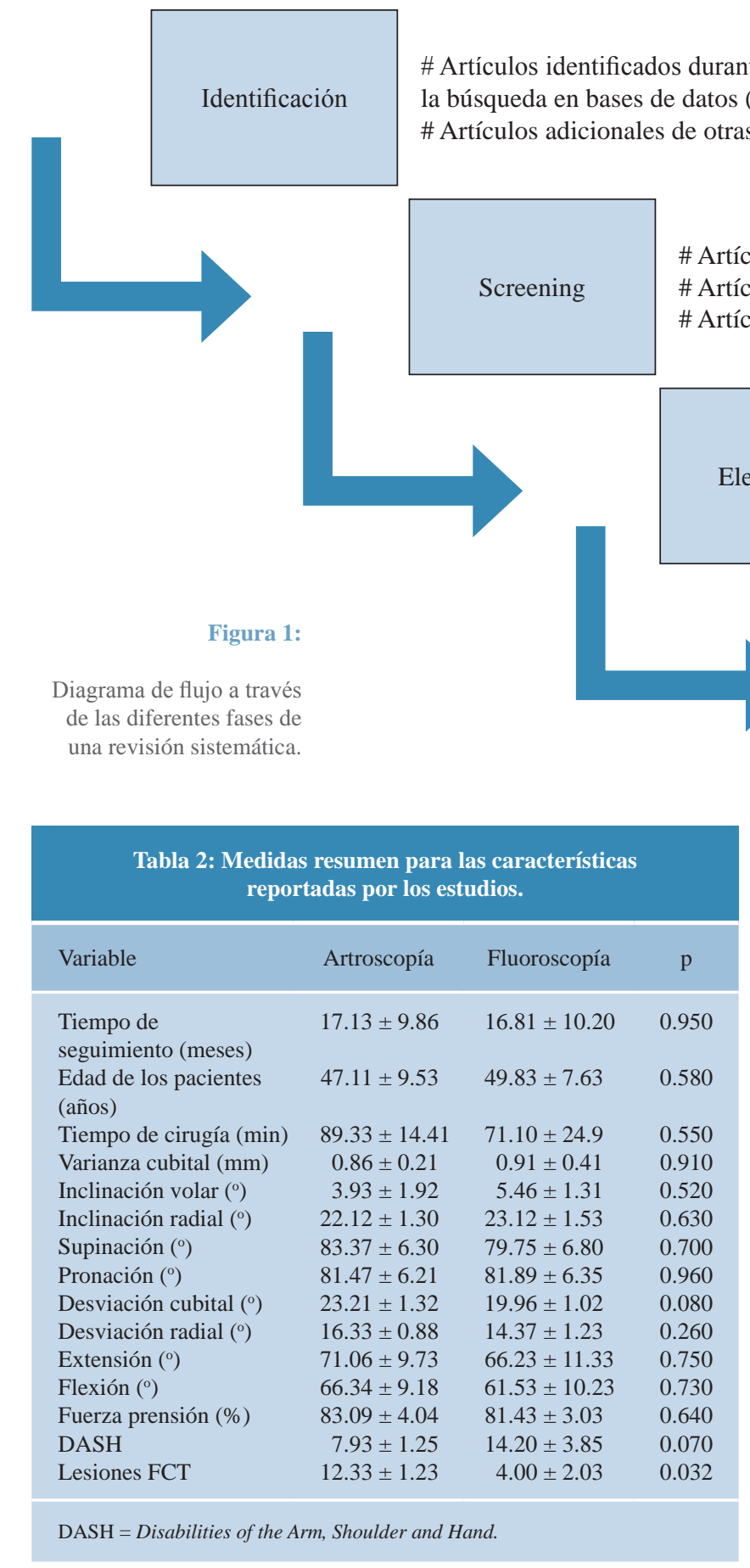

En cuanto al desarrollo de artrosis a largo plazo, Kordasiewicz y colaboradore ${ }^{16}$ describieron cuatro casos en artroscopía y seis casos en fluoroscopía, todos de grado I por escalones entre 1 y $2 \mathrm{~mm}$. Doi y su equipo ${ }^{14}$ presentaron 13 casos de artrosis grado I y tres casos grado II en artroscopía con respecto a 12 casos de grado I; 12 de grado II y cuatro grado III encontrados en el grupo de fluoroscopía.

Respecto al escore DASH, éste presentó correlación significativa con la edad promedio de los pacientes $(\mathrm{R}=0.78$, $\mathrm{p}=0.002)$ y con extensión, aunque de manera diferencial:

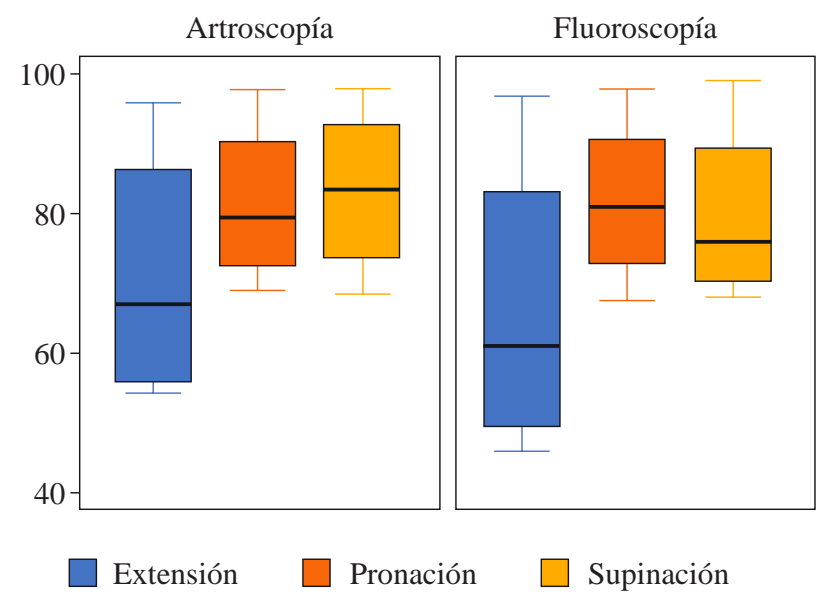

Figura 2: Diagrama de cajas para extensión, pronación y supinación en función del tratamiento.

sólo fue indirecta en fluoroscopía ( $\mathrm{R}=-0.34, \mathrm{p}=0.042)$. La Figura 4 ilustra dichas asociaciones y en promedio para ambos tratamientos.

La fuerza de prensión no se correlacionó con el tiempo de seguimiento $(\mathrm{p}=0.312)$ (Figura 5). Cuando la asociación entre esta variable (fuerza de prensión) y flexión se analizó por tratamiento, sólo mostró correlación directa y significativa en los grupos de pacientes tratados con fluoroscopía $(\mathrm{R}=0.97, \mathrm{p}=0.003$ versus $\mathrm{R}=0.14, \mathrm{p}=0.74$, mediante artroscopía).

\section{Discusión}

En 2010 la Academia Americana de Cirujanos Ortopedistas (AAOS American Academy of Orthopaedics Surgeons) 
publicó una guía de práctica clínica para el tratamiento de fracturas de radio distal basada en una revisión sistemática rigurosa de la bibliografía, los autores concluyeron que la evidencia en relación a la evaluación artroscópica de la superficie articular en fracturas intraarticulares de radio distal era escasa, por lo que el grado de recomendación a favor de realizar artroscopía era débil. ${ }^{2}$

En esta revisión sistemática se encontraron siete estudios que comparan la reducción de fracturas de radio distal intraarticular bajo fluoroscopía versus asistencia artroscópica. En general los resultados de las características analizadas fueron homogéneos, cabe destacar que se encontraron datos a favor de la asistencia artroscópica, con diferencias estadísticamente significativas a favor de este tratamiento en la escala de DASH, desviación cubital y el diagnóstico de lesiones del CFCT.

Sólo dos trabajos ${ }^{14,16}$ analizan la variable artrosis, por lo que no fue posible realizar un análisis estadístico de la relación entre esta característica, incongruencia articular y resultados funcionales. Doi y colaboradores ${ }^{14}$ encontraron asociación entre el análisis de incongruencia articular y grado de artrosis con resultados favorables en el grupo de artroscopía, pero no establecieron asociación entre los resultados de las escalas funcionales y artrosis. Kordasiewicz y su equipo $^{16}$ no observaron correlación entre artrosis y resultados clínicos en ambos grupos, pero destacan que la asistencia artroscópica en fracturas B1 de la clasificación AO logra identificar y corregir incongruencias que pasan desapercibidas sólo utilizando asistencia fluoroscópica, sus resultados se ven reflejados en la ausencia de artrosis en estos pacientes en comparación con el grupo fluoroscopía. Se sabe que el desarrollo de osteoartrosis a nivel de miembro superior es un proceso de progresión lenta ${ }^{4,22,23}$ y es frecuente que la clínica no se correlacione con los resultados radiográficos finales. ${ }^{23}$ Catalano y colaboradores ${ }^{4}$ y Fernandez y su equipo $^{24}$ demostraron la asociación entre escalones articulares residuales y el desarrollo de artrosis, pero a pesar de esto la
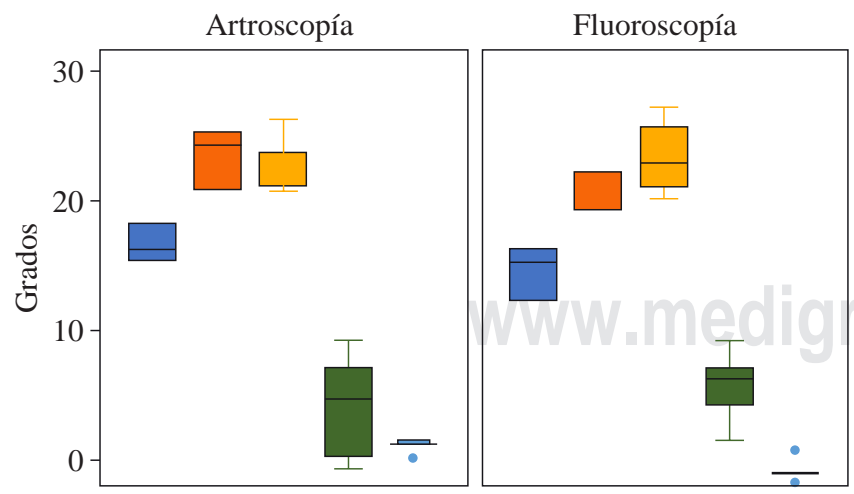

Desviación radial

Inclinación volar

Desviación cubital

Inclinación radial

Figura 3: Diagrama de cajas para desviación radial, cubital, inclinación radial, volar y variación cubital en función del tratamiento.

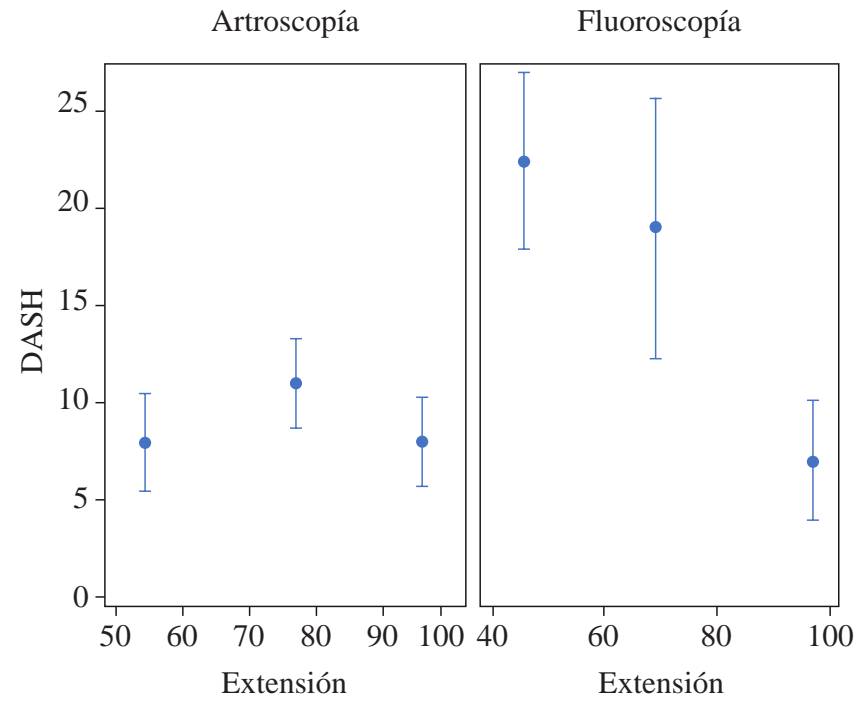

DASH $=$ Disabilities of the Arm, Shoulder and Hand.

Figura 4: Distribuciones del escore Disabilities of the Arm, Shoulder and Hand en función de los promedios de extensión logrados en los grupos de pacientes.

presencia de artrosis no se correlaciona con los resultados funcionales. En los siete trabajos evaluados el tiempo de seguimiento promedio fue de 15.98 meses (rango de tres a 31 meses), tiempo corto para establecer correlaciones entre artrosis y resultados funcionales.

No se observó consenso en la elección de las escalas de evaluación funcional. Sólo cuatro estudios utilizaron la escala de DASH para evaluar función. Varitimidis y colaboradores ${ }^{11}$ encontraron diferencias significativas a los tres meses a favor del grupo bajo asistencia artroscópica, pero al evaluar el mismo grupo al año y a los dos años el resultado no varió. Yamazaki, ${ }^{18} \mathrm{Ruch}^{12}$ y Kordasiewicz ${ }^{16}$ registraron datos similares. Doi y colegas ${ }^{14}$ presentaron resultados a favor del grupo artroscopía utilizando la Escala de Green y O'Brien modificado por Cooney y la clasificación funcional de Garltland y Werley. Lalone ${ }^{17}$ registró mejores resultados en el mismo grupo utilizando la escala PRWE. Estos resultados son cuestionables, no analizan relación entre resultados funcionales y severidad de fractura, ni tipo de osteosíntesis. En estos trabajos la elección del material de osteosíntesis fue heterogénea, algunos autore ${ }^{17}$ utilizaron placas dorsales, material asociado a menor desempeño funcional. ${ }^{25}$

Es frecuente encontrar lesiones ligamentarias asociadas a fracturas de radio distal, pero todavía es controvertido cómo su presencia afecta los resultados y cuál es la mejor manera de abordarlas. Las datos reportados de lesiones ligamentarias asociadas se extienden entre 18 y $86 \%$ para lesiones del ligamento escafolunar, ${ }^{26,27}$ de $5 \%$ a $29 \%$ para el ligamento lunopiramidal ${ }^{27,28}$ y de 17 a $60 \%$ para el CFCT. ${ }^{11,29}$ Es incierta la incidencia de lesiones de los ligamentos del carpo evaluados sólo por fluoroscopía ${ }^{2}$ y las lesiones del CFCT pueden pasar desapercibidas ante la ausencia de inestabilidad radio cubital distal. La artroscopía es el método de diag- 
nóstico estándar de oro para identificar estas lesiones, los resultados obtenidos en este metaanálisis corroboran estos datos. A pesar de esto, no existen investigaciones que a largo plazo nos permitan identificar cuáles son sintomáticas y deben ser tratadas en este contexto. ${ }^{30}$ Es frecuente observar en radiografías de fracturas de radio distal distasis del espacio escafosemilunar mayor de $3 \mathrm{~mm} ; ;^{31,32,33}$ sin embargo, no siempre es clínicamente relevante, tal vez debido a la cicatrización ligamentaria durante la inmovilización. 34,35,36,37

La artroscopía se ha convertido en una herramienta de uso frecuente para diagnóstico y tratamiento de patología de muñeca entre los cirujanos de miembro superior, es importante destacar que la curva de aprendizaje es exigente. Obdeijn y colaboradores..$^{38}$ analizaron las tendencias en artroscopía de muñeca a través de una encuesta aplicada a cirujanos de mano. Para ser considerado un experto en esta técnica, la cantidad mínima de artroscopías realizadas debe ser 50 y para mantener la habilidad entre 20 y 30 por año.

Se encontraron como limitaciones de este trabajo el número de artículos analizados, la heterogeneidad de datos, la escasez de los mismos al analizar algunas variables y la falta de consenso entre los autores de los artículos para elegir métodos de evaluación funcional. No fue posible llegar a conclusiones en relación a la hipótesis planteada. Sólo dos artículos evaluaron la asociación entre las variables incongruencia articular, desarrollo de artrosis y resultados funcionales. No fue posible establecer en qué casos es recomendable realizar evaluación artroscópica.

\section{Conclusión}

Siete años después de la publicación realizada por el grupo de la AAOS, ${ }^{2}$ los trabajos científicos encontrados en esta

Artroscopía $\quad$ Fluoroscopía

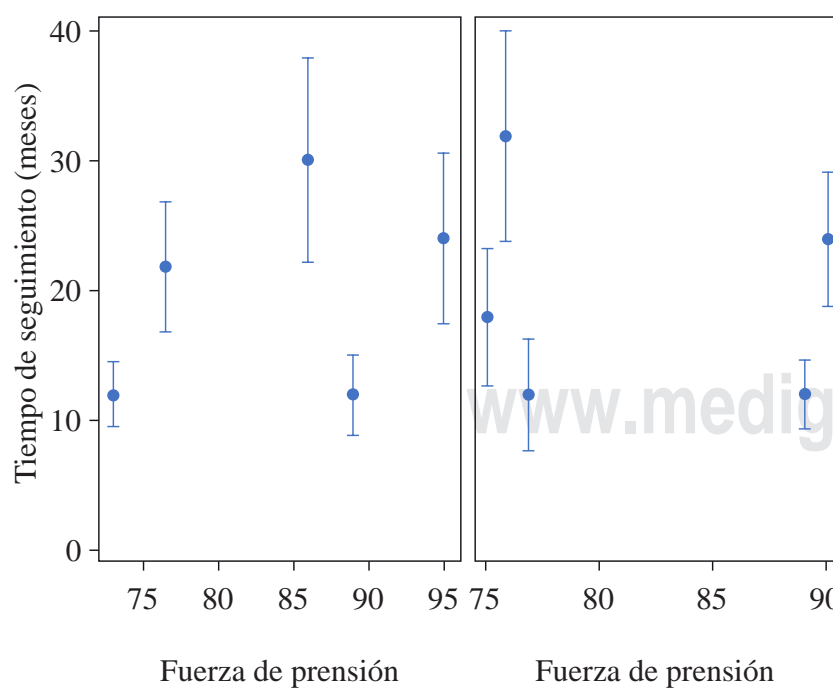

Figura 5: Distribuciones del tiempo de seguimiento (en meses) en función de los promedios de la fuerza de prensión obtenidas en los grupos de pacientes. revisión sistemática no superan el Nivel de evidencia II+, lo que significa que la evidencia disponible es controvertida y no permite hacer recomendaciones a favor o en contra de la intervención, otros factores podrían influir en la decisión.

\section{Referencias}

1. Herzberg G. Intra-articular fracture of the distal radius: arthroscopicassisted reduction. J Hand Surg Am. 2010; 35(9): 1517-9.

2. Lichtman DM, Bindra RR, Boyer MI, Putnam MD, Ring D, Slutsky DJ, et al. Treatment of distal radius fractures. J Am Acad Orthop Surg. 2010; 18(3): 180-9.

3. Knirk JL, Jupiter JB. Intra-articular fractures of the distal end of the radius in young adults. J Bone Joint Surg Am. 1986; 68: 647-59.

4. Catalano LW III, Cole RJ, Gelberman RH, Evanoff BA, et al. Displaced intra-articular fractures of the distal aspect of the radius. Long-term results in young adults after open reduction and internal fixation. J Bone Joint Surg. 1997; 79A: 1290-1302.

5. Goldfarb CA, Rudzki JR, Catalano LW, Hughes M, Borrelli J Jr. Fifteen-year outcome of displaced intra-articular fractures of the distal radius. J Hand Surg Am. 2006; 31(4): 633-9.

6. Haus BM, Jupiter JB. Intra-articular fractures of the distal end of the radius in young adults: reexamined as evidence-based and outcomes medicine. J Bone Joint Surg Am. 2009; 91(12): 2984-91. doi: 10.2106/ JBJS.I.00269.

7. Medoff RJ. Essential radiographic evaluation for distal radius fractures. Hand Clin. 2005; 21: 279-88.

8. Dario P, Matteo G, Carolina C, Marco G, Cristina D, Daniele F, et al. Is it really necessary to restore radial anatomic parameters after distal radius fractures? Injury. 2014; 45(Suppl 6): S21-6. doi: 10.1016/j. injury.2014.10.018.

9. Whipple TL. Intraarticular fractures of the distal radius and carpals. In: Whipple TL, editor. Arthroscopic surgery-the wrist. Philadelphia: JB Lippincott Company; 1992. 143-8.

10. Duncan SF, Weiland Minimally invasive reduction and osteosynthesis of articular fractures of the distal radius. Injury. 2001; 32(Suppl 1): SA14-24.

11. Varitimidis SE, Basdekis GK, Dailiana ZH, Hantes ME, Bargiotas K, Malizos K. Treatment of intra-articular fractures of the distal radius: fluoroscopic or arthroscopic reduction? J Bone Joint Surg Br. 2008; 90: 778-85.

12. Ruch DS, Vallee J, Poehling GG, Smith BP, Kuzma GR. Arthroscopic reduction versus fluoroscopic reduction in the management of intraarticular distal radius fractures. Arthroscopy. 2004; 20: 225-30.

13. Edwards CC, Haraszti CJ, McGillivary GR, Gutow AP. Intra-articular distal radius fractures: arthroscopic assessment of radiographically assisted reduction. J Hand Surg. 2001; 26A: 1036-41. 03635023/01/26A06-0038\$35.00/0. doi: 10.1053/jhsu.2001.28760.

14. Doi K, Hattori Y, Otsuka K, Abe Y, Yamamoto H. Intra-articular fractures of the distal aspect of the radius: arthroscopically assisted reduction compared with open reduction and internal fixation. $J$ Bone Joint Surg. 1999; 81(8): 1093-10.

15. Hutton B, Catalá-López F, Mohera D. La extensión de la declaración PRISMA para revisiones sistemáticas que incorporan metaanálisis en red: PRISMA-NMA. Med Clin (Barc). 2016; 147(6): 262-6.

16. Kordasiewicz B, Podgórski A, Klich M, Michalik D, Chaberek S, Pomianowski S. Arthroscopic assessment of intraarticular distal radius fractures results of minimally invasive fixation. Ortop Traumatol Rehabil. 2011; 13: 369-86.

17. Lalone EA, Rajgopal V, Roth J, Grewal R, MacDermid JC. A cohort study of one-year functional and radiographic outcomes following intra-articular distal radius fractures. Hand (NY). 2014; 9: 237-43.

18. Yamazaki H, Uchiyama S, Komatsu M, Hashimoto S, Kobayashi Y, Sakurai T, Kato H Arthroscopic assistance does not improve the functional or radiographic outcome of unstable intra-articular distal radial fractures treated with a volar locking plate: a randomised controlled trial. Bone Joint J. 2015; 97(7): 957-962.

19. Christiaens N, Nedellec G, Guerre E, Guillou J, Demondion X, Fontain C, et al. Contribution of arthroscopy to the treatment of 
intraarticular fracture of the distal radius: Retrospective study of 40 cases. Hand Surg Rehab Hand Surg Rehabil. 2017; 36(4): 268-74. doi: 10.1016/j.hansur.2017.03.003.

20. Fernandez DL, Jupiter JB. Fractures of the distal radius. A practical approach to management. New York, Springer-Verlag, 1996.

21. Kennedy CA, Beaton DE, Solway S, McConnell S, Bombardier C. Disabilities of the Arm, Shoulder and Hand (DASH). The DASH and QuickDASH Outcome Measure User's Manual. 3rd ed. Toronto, Ontario: Institute for Work \& Health; 2011.

22. Viegas SF, Patterson RM. Load mechanics of the wrist. Hand Clin. 2015; 13: 109-28.

23. Lutz M, Arora R, Krappinger D, Wambacher M, Rieger M, Pechlaner S. Arthritis predicting factors in distal intraarticular radius fractures. Arch Orthop Trauma Surg. 2011; 131(8): 1121-6. doi: 10.1007/ s00402-010-1211-3.

24. Fernandez JJ, Gruen GS, Herndon JH. Outcome of distal radius fractures using the short form 36 health survey. Clin Orthop Relat Res. 1997; 341: 36-41.

25. Ruch DS, Papadonikolakis A. Volar versus dorsal plating in the management of intra- articular distal radius fractures. J Hand Surg [Am]. 2006; 31: 9-16.

26. Shih JT, Lee HM, Hou YT, Tan CM. Arthroscopically-assisted reduction of intra- articular fractures and soft tissue management of distal radius. Hand Surg. 2001; 6(2): 127-35.

27. Forward DP, Lindau TR, Melsom DS. Intercarpal ligament injuries associated with fractures of the distal part of the radius. J Bone Joint Surg Am. 2007; 89(11): 2334-40.

28. Espinosa-Gutiérrez A, Rivas-Montero JA, Elias-Escobedo A, AlisedoOchoa PG. Wrist arthroscopy for fractures of the distal end of the radius. Acta Ortop Mex. 2009; 23(6): 358-65.
29. Hardy P, Gomes N, Chebil M, Bauer T. Wrist arthroscopy and intraarticular fractures of the distal radius in young adults. Knee Surg Sports Traumatol Arthrosc. 2006; 14(11): 1225-30.

30. Swart E, Tang P. The effect of ligament injuries on outcomes of operatively treated distal radius fractures. Am J Orthop (Belle Mead NJ). 2017; 46(1): E41-6.

31. Rosenthal DI, Schwartz M, Phillips WC, Jupiter J. Fracture of the radius with instability of the wrist. AJR Am J Roentgenol. 1983; 141(1): 113-6.

32. Jones VM, Everding NG, Desmarais JM, Soong MC. Scapholunate instability after distal radius volar plating. Hand (NY). 2015; 10(4): 678-82.

33. Tang JB, Shi D, Gu YQ, Zhang QG. Can cast immobilization successfully treat scapholunate dissociation associated with distal radius fractures? J Hand Surg Am. 1996; 21(4): 583-90.

34. Gunal I, Ozaksoy D, Altay T, Satoglu IS, Kazimoglu C, Sener M. Scapholunate dissociation associated with distal radius fractures. Eur J Orthop Surg Traumatol. 2013; 23(8): 877-81.

35. Bunker DL, Pappas G, Moradi P, Dowd MB. Radiographic signs of static carpal instability with distal end radius fractures: Is current treatment adequate? Hand Surg. 2012; 17(3): 325-30.

36. Desai MJ, Kamal RN, Richard MJ. Management of intercarpal ligament injuries associated with distal radius fractures. Hand Clin. 2015; 31(3): 409-16.

37. Lans J, Lasa A, Chen NC, Jupiter JB. Incidence and functional outcomes of scapholunate diastases associated distal radius fractures: a 2-year follow-up scapholunate dissociation. Open Orthop J. 2018; 12: 33-40. doi: 10.2174/1874325001812010033.

38. Obdeijn MC, Tuijthof GJ, Van der Horst CM, Mathoulin C, Liverneaux P. Trends in wrist arthroscopy. J Wrist Surg. 2013; 2(3): 239-46. doi: 10.1055/s-0033-1351355. 